\title{
Sur la proximité des nombres puissants
}

\author{
par
}

\section{Jean-Marie De Koninck (Québec) et Florian Luca (Morelia)}

1. Introduction. On dit qu'un entier $n>1$ est un nombre puissant si pour chaque nombre premier $p$ qui divise $n$, on a $p^{2} \mid n$. La distribution des nombres puissants a été largement étudiée. En particulier, on sait que si $S(N)$ désigne le nombre de nombres puissants qui n'excèdent pas $N$, alors

$$
S(N)=C_{1} \sqrt{N}+O\left(N^{1 / 3}\right), \quad C_{1}=\frac{\zeta(3 / 2)}{\zeta(3)} \approx 2.1732,
$$

où $\zeta$ désigne la fonction zêta de Riemann. Des estimations encore plus précises sont connues (voir, par exemple, le théorème 14.4 du livre de Ivić [1]). Comme la constante $C_{1}$ apparaissant dans (1) est supérieure à 2 , il existe une infinité d'entiers positifs $n$ tels que l'intervalle $] n^{2},(n+1)^{2}$ [ admet au moins deux nombres puissants.

Nous démontrons ici que quel que soit l'entier positif $k$, il existe une infinité d'entiers positifs $n$ tels que l'intervalle $] n^{2},(n+1)^{2}$ [ contient au moins $k$ nombres puissants. Nous établissons également que l'ensemble des entiers positifs $n$, dont l'intervalle $] n^{2},(n+1)^{2}$ [ correspondant ne contient aucun nombre puissant, admet une densité, et nous calculons sa valeur.

Dans cet article, nous utilisons les symboles de Landau $O$ et $o$ de même que le symbole de Vinogradov $\ll$ avec leurs significations habituelles.

\section{Les principaux résultats}

THÉORÈME 1. Il existe une infinité d'entiers positifs n tels que l'intervalle ]$n^{2},(n+1)^{2}[$ contient plus de

$$
\frac{9}{20}\left(\frac{\log n}{\log \log n}\right)^{1 / 3}
$$

nombres puissants.

2000 Mathematics Subject Classification: 11A51, 11J13.

Travail de J.-M. De Koninck soutenu en partie par une subvention du CRSNG.

Travail de F. Luca soutenu en partie par les projets SEP-CONACYT 37259-E et 37260-E. 
ThÉorème 2. Si $V(N)$ désigne le nombre d'entiers positifs $n \leq N$ tels que l'intervalle $] n^{2},(n+1)^{2}$ [ ne contient aucun nombre puissant, alors

$$
V(N)=C_{2} N+O\left(\frac{N}{\sqrt{\log \log N}}\right),
$$

où

$$
C_{2}=\prod_{m=2}^{\infty}\left(1-\frac{\mu^{2}(m)}{m^{3 / 2}}\right) \approx 0.275 .
$$

3. La preuve du théorème 1. Soit $k$ un entier positif grand mais fixe et $\alpha_{1}, \ldots, \alpha_{2 k}$ des nombres irrationnels positifs que l'on choisira plus tard. Selon le théorème de Dirichlet relatif aux approximations diophantiennes simultanées, pour tout nombre réel $Q>1$, il existe un entier positif $q \leq Q$ et des entiers $p_{1}, \ldots, p_{2 k}$ tels que les inégalités

$$
\left|\alpha_{j}-\frac{p_{j}}{q}\right| \leq \frac{1}{q Q^{1 / 2 k}} \quad(j=1, \ldots, 2 k)
$$

sont satisfaites. Soit par ailleurs $q_{1}<\ldots<q_{n}<\ldots$ la suite infinie d'entiers positifs telle que pour chaque $n \geq 1$, il existe des entiers $p_{n, 1}, \ldots, p_{n, 2 k}$ tels que les inégalités

$$
\left|\alpha_{j}-\frac{p_{n, j}}{q_{n}}\right| \leq \frac{1}{q_{n}^{1+1 / 2 k}} \quad(j=1, \ldots, 2 k)
$$

sont satisfaites. Le fait que $\left(q_{n}\right)_{n \geq 1}$ est une suite infinie découle de la construction des inégalités (2) en tenant compte que les nombres $\alpha_{j}$, pour $j=1, \ldots, 2 k$, sont irrationnels. Supposons maintenant que $c_{1} \geq 1$ est une constante telle que les inégalités

$$
\left|\alpha_{j}-\frac{p}{q}\right|>\frac{1}{c_{1} q^{2}} \quad(j=1, \ldots, 2 k)
$$

sont satisfaites pour tous les entiers $p$ et $q$ avec $q \neq 0$. Tel est le cas par exemple lorsque les réduites de la fraction continue de chaque $\alpha_{j}$ sont bornées par $c_{1}-1$.

En utilisant (4), on obtient aisément que pour $n \geq 1$, en posant $Q_{n}=$ $\left(c_{1} q_{n}\right)^{4 k}$ et en utilisant (2), il existe des entiers $p_{1}, \ldots, p_{2 k}, q$ avec $1 \leq q \leq Q_{n}$ pour lesquels les inégalités (2) sont vérifiées avec $Q_{n}$ à la place de $Q$. On a ainsi établi que les inégalités

$$
\left|\alpha_{j}-\frac{p_{j}}{q}\right| \leq \frac{1}{q Q_{n}^{1 / 2 k}} \leq \frac{1}{Q_{n}^{1 / 2 k}}=\frac{1}{\left(c_{1} q_{n}\right)^{2}} \leq \frac{1}{c_{1} q_{l}^{2}}<\left|\alpha_{j}-\frac{p_{l, j}}{q_{l}}\right|
$$

tiennent pour $j=1, \ldots, 2 k$ et $l=1, \ldots, n$, et en particulier que $q \neq q_{l}$ pour $l=1, \ldots, n$. Ainsi, $q \geq q_{n+1}$, et on a donc démontré que

$$
q_{n+1} \leq\left(c_{1} q_{n}\right)^{4 k} \quad(n=1,2, \ldots) .
$$


Pour chaque entier $j \geq 1$, soit $m_{j}$ le $j$-ième entier positif tel que $m_{j}^{2}+1$ est libre de carrés, et pour $j=1, \ldots, 2 k$, posons $d_{j}=m_{j}^{2}+1$. Avec un tel choix des $d_{j}$, il est alors clair que les nombres $d_{j} p_{j}^{2}, j=1, \ldots, 2 k$, sont tous distincts. De plus, d'après un résultat de Ricci [3], étant donné un polynôme primitif irréductible $f(x)$ de degré $r \geq 2$ à coefficients entiers, l'ensemble $\left\{m \geq 1: p^{i} \mid f(m) \Rightarrow i<r\right\}$ est de densité positive. En particulier, l'ensemble $\left\{m \geq 1: \mu^{2}\left(m^{2}+1\right)=1\right\}$ est de densité positive, et il est facile de voir que cette densité est égale à

$$
\prod_{p \equiv 1 \bmod 4}\left(1-\frac{2}{p^{2}}\right) \approx 0.89>\frac{1}{2},
$$

de sorte que si $j$ est suffisamment grand, $d_{j}=m_{j}^{2}+1<(2 j)^{2}+1$, auquel cas $d_{j}<4 j^{2}$. Il s'ensuit qu'en posant $D:=\prod_{j=1}^{2 k} d_{j}$, on a $D<\left(16 k^{2}\right)^{2 k}$.

Pour chaque $j=1, \ldots, 2 k$, posons maintenant $\alpha_{j}=1 / \sqrt{d_{j}}$. Ainsi choisi, chaque nombre $\alpha_{j}$ est irrationnel, et son développement en fraction continue est $\alpha_{j}=\left[0, m_{j}, 2 m_{j}, 2 m_{j}, \ldots\right]$. Cela veut dire que l'on doit prendre $c_{1}$ supérieur à $\max \left\{2 m_{j}: j=1,2, \ldots, 2 k\right\}$. Or, comme $m_{j}<2 j$ pour $j$ suffisamment grand, il s'avère qu'en choisissant $c_{1}=8 k$, l'inégalité (4) est satisfaite.

Soit maintenant $q=q_{m}$ pour un certain $m$ que l'on choisira plus tard, et soit $p_{j}=p_{m, j}$ pour chaque entier $j=1, \ldots, 2 k$. Fixons $j$. L'inégalité

$$
\left|\alpha_{j}-\frac{p_{j}}{q}\right| \leq \frac{1}{q^{1+1 / 2 k}}
$$

entraîne que

$$
\left|q-\sqrt{d_{j}} p_{j}\right| \leq \sqrt{d_{j}} q^{-1 / 2 k}
$$

Supposons que

$$
q>d_{j}^{k}
$$

Dans ce cas, l'inégalité (5) implique que $\sqrt{d_{j}}\left|p_{j}\right|<q+1$, et donc que $\left|q+\sqrt{d_{j}} p_{j}\right|<2 q+1<3 q$, ce qui entraîne que

$$
\left|q^{2}-d_{j} p_{j}^{2}\right|=\left|q-\sqrt{d_{j}} p_{j}\right| \cdot\left|q+\sqrt{d_{j}} p_{j}\right|<3 \sqrt{d_{j}} q^{1-1 / 2 k} .
$$

Puisque, pour $j=1, \ldots, 2 k, \sqrt{d_{j}}<\left(16 k^{2}+1\right)^{1 / 2}$, on a alors les inégalités

$$
\left|(D q)^{2}-d_{j} D^{2} p_{j}^{2}\right|<3 D^{2}\left(16 k^{2}+1\right)^{1 / 2} q^{1-1 / 2 k} \quad(j=1, \ldots, 2 k) .
$$

Posons maintenant $n=D q$ et choisissons $k$ de telle manière que

$$
3 D^{2}\left(16 k^{2}+1\right)^{1 / 2} q^{1-1 / 2 k}<2(n-1)+1=2 D q-1 .
$$

Ainsi, afin que (9) tienne, il suffit de choisir $q$ de telle sorte que

$$
3 D\left(16 k^{2}+1\right)^{1 / 2} q^{1-1 / 2 k}<q,
$$


ce qui revient à écrire

$$
q>\left(3\left(16 k^{2}+1\right)^{1 / 2} D\right)^{2 k} .
$$

Observons que (10) entraîne (6).

Puisque les nombres $d_{j} D^{2} p_{j}^{2}, j=1, \ldots, 2 k$, sont tous distincts (ceci découlant du fait que les $d_{j} p_{j}^{2}$ sont distincts), il découle des inégalités (8) et (9) qu'au moins $k$ des nombres $d_{j} D^{2} p_{j}^{2}$ appartiennent soit à l'intervalle ] $(n-1)^{2}, n^{2}$ [, soit à l'intervalle $] n^{2},(n+1)^{2}$ [, et il est clair par ailleurs qu'ils sont tous des nombres puissants. Ainsi, il suffit maintenant de choisir $q=q_{m}$ comme étant le plus petit nombre satisfaisant l'inégalité (10). Un tel nombre va aussi satisfaire les inégalités

$$
\begin{aligned}
q & <\left(c_{1}\left(3\left(16 k^{2}+1\right)^{1 / 2} D\right)^{2 k}\right)^{4 k}<\exp \left\{4 k \log \left(c_{1}\left(3 \sqrt{16 k^{2}+1}\left(16 k^{2}\right)^{2 k}\right)^{2 k}\right)\right\} \\
& \leq \exp \left\{8 k^{2} \cdot 2 k \cdot(1+o(1)) \cdot \log \left(16 k^{2}\right)\right\}=\exp \left\{32(1+o(1)) k^{3} \log k\right\},
\end{aligned}
$$

où nous avons utilisé le fait que $\left(16 k^{2}+1\right)^{1 / 2}<c_{1}=8 k$.

Comme $q D=n$ et comme

$$
D<\left(16 k^{2}\right)^{2 k}=\exp \left\{2 k \log \left(16 k^{2}\right)\right\}=\exp \{4 k(1+o(1)) \log k\},
$$

on a

$$
n=q D<\exp \left\{32(1+o(1)) k^{3} \log k\right\} .
$$

On obtient ainsi

$$
32(1+o(1)) k^{3} \log k \geq \log n,
$$

de sorte que

ce qui montre que

$$
\frac{32}{3} k^{3} \log \left(k^{3}\right) \geq(1+o(1)) \log n,
$$

$$
k \geq\left(\frac{3}{32}\right)^{1 / 3}(1+o(1))\left(\frac{\log n}{\log \log n}\right)^{1 / 3},
$$

laquelle inégalité entraîne certainement que

$$
k \geq \frac{9}{20}\left(\frac{\log n}{\log \log n}\right)^{1 / 3},
$$

pour $n$ suffisamment grand, puisque $(3 / 32)^{1 / 3}>9 / 20$. Voilà qui complète la preuve du théorème 1 .

4. La preuve du théorème 2. Soit $N$ un grand nombre réel positif. Soit $n \leq N$ et supposons que l'intervalle $] n^{2},(n+1)^{2}$ [ contient effectivement un nombre puissant. On peut alors écrire ce nombre puissant de manière unique sous la forme $m^{3} l^{2}$, où $m$ est un nombre libre de carrés. Il est clair qu'on a alors

$$
n=\lfloor m \sqrt{m} \cdot l\rfloor,
$$


alors que réciproquement si (11) est satisfaite pour un certain nombre libre de carrés $m$ et un certain entier positif $l$, le nombre puissant correspondant $m^{3} l^{2}$ est situé dans l'intervalle $] n^{2},(n+1)^{2}$ [. C'est pourquoi il suffit de compter le nombre de nombres $n \leq N$ satisfaisant (11) pour un certain nombre $m$ libre de carrés et un certain entier positif $l$.

Pour chaque nombre $m$ libre de carrés, posons

$$
\mathcal{A}_{m}=\{\lfloor m \sqrt{m} \cdot l\rfloor \leq N: l \geq 1\} .
$$

Il nous faut estimer la cardinalité de l'ensemble

$$
\mathcal{A}=\bigcup_{\substack{m \geq 1 \\ \mu(m) \neq 0}} \mathcal{A}_{m}
$$

Soit $Y$ un paramètre réel qui tend vers l'infini avec $N$, d'une manière que l'on indiquera plus tard. Observons que

et ainsi que

$$
\# \mathcal{A}_{m} \ll \frac{N}{m^{3 / 2}}
$$

$$
\# \bigcup_{m>Y} \mathcal{A}_{m} \leq \sum_{m>Y} \# \mathcal{A}_{m} \ll N \sum_{m>Y} \frac{1}{m^{3 / 2}} \ll N \int_{Y}^{\infty} \frac{d t}{t^{3 / 2}} \ll \frac{N}{Y^{1 / 2}} .
$$

Pour $m \leq Y$, en utilisant le principe d'inclusion-exclusion, nous obtenons

$$
\# \bigcup_{\substack{1<m \leq Y \\ \mu(m) \neq 0}} \mathcal{A}_{m}=\sum_{j \geq 1}(-1)^{j-1} \sum_{\substack{1<m_{1}<\ldots<m_{j} \leq Y \\ \mu\left(m_{i}\right) \neq 0, i=1, \ldots, j}} \# \bigcap_{i=1}^{j} \mathcal{A}_{m_{i}} .
$$

Fixons $j$ et soit $1<m_{1}<\ldots<m_{j} \leq Y$ des nombres libres de carrés. Posons $\alpha_{i}=m_{i}^{3 / 2}$ et $\beta_{i}=1 / \alpha_{i}$ pour $i=1, \ldots, j$. Soit $n \in \bigcap_{i=1}^{j} \mathcal{A}_{m_{i}}$. Il s'ensuit qu'il existe des entiers $l_{i}$, pour $i=1, \ldots, j$, tels que les inégalités $n<\alpha_{i} l_{i}<n+1$ sont satisfaites pour chaque $i=1, \ldots, j$. Or, ces dernières inégalités sont équivalentes au fait que les inégalités $n \beta_{i}<l_{i}<n \beta_{i}+\beta_{i}$ tiennent aussi pour $i=1, \ldots, j$. Il s'ensuit que $\left\{n \beta_{i}\right\} \in I_{i}$ pour $i=1, \ldots, j$, où pour chaque nombre $i$, l'expression $I_{i}$ désigne l'intervalle $\left(1-\beta_{i}, 1\right)$. Ici, $\{x\}$ désigne la partie fractionnaire de $x$. Observons que la longueur de $I_{i}$ est $\left|I_{i}\right|=\beta_{i}$ pour chaque $i=1, \ldots, j$.

Il est clair que les nombres $1, \beta_{1}, \ldots, \beta_{j}$ sont linéairement indépendants sur $\mathbb{Q}$. En fait, les nombres 1 et $m^{-3 / 2}$, pour chaque entier $m>1$ libre de carrés, sont linéairement indépendants sur $\mathbb{Q}$ et, en réalité, forment une base pour le corps $\mathbb{Q}[\sqrt{p}: p$ premier $]$, en tant qu'espace vectoriel sur $\mathbb{Q}$.

Pour chaque entier positif $n \leq N$, posons $\mathbf{x}_{n}:=\left(\left\{n \beta_{1}\right\}, \ldots,\left\{n \beta_{j}\right\}\right)$. Il découle de la théorie de la distribution uniforme des suites multidimensionnelles modulo 1 (voir le chapitre 2 de Kuipers et Niederreiter [2]) que 


$$
\bigcap_{i=1}^{j} \mathcal{A}_{m_{i}}=N \prod_{i=1}^{j} \beta_{i}+o(N),
$$

où le terme d'erreur dans (15) est

$$
\leq N D_{\beta_{1}, \ldots, \beta_{j}, N}\left(\mathbf{x}_{1}, \ldots, \mathbf{x}_{N}\right)
$$

la quantité $D_{\beta_{1}, \ldots, \beta_{j}, N}\left(\mathbf{x}_{1}, \ldots, \mathbf{x}_{N}\right)$ désignant la discrépance de nos suites (de dimensions $j)\left(\mathbf{x}_{1}, \ldots, \mathbf{x}_{N}\right)$. Cette discrépance satisfait l'inégalité

$$
\begin{aligned}
& D_{\beta_{1}, \ldots, \beta_{j}, N}\left(\mathbf{x}_{1}, \ldots, \mathbf{x}_{N}\right) \\
& \leq 2 j^{2} 3^{j+1}\left(\frac{1}{M}+\sum_{0<\|\mathbf{h}\| \leq M} \frac{1}{r(\mathbf{h})}\left|\frac{1}{N} \sum_{n=1}^{N} e^{2 \pi i\left\langle\mathbf{h}, \mathbf{x}_{n}\right\rangle}\right|\right) .
\end{aligned}
$$

Dans cette dernière inégalité, les notations sont celles utilisées dans le livre de Kuipers et Niederreiter [2], et comme tel, $\mathbf{h}=\left(h_{1}, \ldots, h_{j}\right)$ est un point à coordonnées entières dans $\mathbb{R}^{j},\|\mathbf{h}\|=\max \left\{\left|h_{i}\right|: i=1, \ldots, j\right\}$,

$$
r(\mathbf{h})=\prod_{i=1}^{j} \max \left\{\left|h_{i}\right|, 1\right\},
$$

et $\left\langle\mathbf{h}, \mathbf{x}_{n}\right\rangle$ désigne le produit scalaire dans $\mathbb{R}^{j}$. Dans l'inégalité (17), $M$ est un entier positif arbitraire, lequel est habituellement choisi de telle manière qu'il minimise la borne supérieure donnée par le membre de droite de (17) sur $D_{\beta_{1}, \ldots, \beta_{j}, N}\left(\mathbf{x}_{1}, \ldots, \mathbf{x}_{N}\right)$.

En faisant appel à (13), (14) et (16), on obtient alors

$$
\begin{aligned}
V(N)= & N-\# \bigcup \mathcal{A}_{m} \\
= & N-N \sum_{j \geq 1} \sum_{\substack{1<m_{1}<\ldots<m_{j} \leq Y \\
\mu\left(m_{i}\right) \neq 0, i=1, \ldots, j}}(-1)^{j-1} \prod_{i=1}^{j} m_{i}^{-3 / 2}+O\left(\frac{N}{Y^{1 / 2}}\right) \\
& +O\left(\sum_{j \geq 1} \sum_{\substack{1<m_{1}<\ldots<m_{j} \leq Y \\
\mu\left(m_{i}\right) \neq 0, i=1, \ldots, j}} N D_{\beta_{1}, \ldots, \beta_{j}, N}\left(\mathbf{x}_{1}, \ldots, \mathbf{x}_{N}\right)\right) \\
= & C_{2} N+O\left(\sum_{j \geq 1} \sum_{\substack{1<m_{1}<\ldots<m_{j}, m_{j}>Y \\
\mu\left(m_{i}\right) \neq 0, i=1, \ldots, j}}^{j} \prod_{i=1}^{j} m_{i}^{-3 / 2}\right)+O\left(\frac{N}{Y^{1 / 2}}\right) \\
& +O\left(\sum_{j \geq 1} \sum_{\substack{1<m_{1}<\ldots<m_{j} \leq Y \\
\mu\left(m_{i}\right) \neq 0, i=1, \ldots, j}} N D_{\beta_{1}, \ldots, \beta_{j}, N}\left(\mathbf{x}_{1}, \ldots, \mathbf{x}_{N}\right)\right) .
\end{aligned}
$$

Du fait que le produit infini

$$
P=\prod_{m \geq 1}\left(1+\frac{\mu^{2}(m)}{m^{3 / 2}}\right)
$$


converge, on voit aisément que

$$
\sum_{j \geq 1} \sum_{\substack{1<m_{1}<\ldots<m_{j}, m_{j}>Y \\ \mu\left(m_{i}\right) \neq 0, i=1, \ldots, j}} \prod_{i=1}^{j} m_{i}^{3 / 2} \leq P \sum_{m \geq Y} m^{-3 / 2} \ll \int_{Y}^{\infty} \frac{d t}{t^{3 / 2}} \ll \frac{1}{Y^{1 / 2}},
$$

de sorte qu'en comparant (19) avec (18), on obtient

$$
\begin{aligned}
N-\# \bigcup \mathcal{A}_{m}= & C_{2} N+O\left(\frac{N}{Y^{1 / 2}}\right) \\
& +O\left(\sum_{j \geq 1} \sum_{\substack{1<m_{1}<\ldots<m_{j} \leq Y \\
\mu\left(m_{i}\right) \neq 0, i=1, \ldots, j}} N D_{\beta_{1}, \ldots, \beta_{j}, N}\left(\mathbf{x}_{1}, \ldots, \mathbf{x}_{N}\right)\right) .
\end{aligned}
$$

Nous estimons maintenant les discrépances $D_{\beta_{1}, \ldots, \beta_{j}, N}\left(\mathbf{x}_{1}, \ldots, \mathbf{x}_{N}\right)$. Soit $\mathbb{K}_{Y}=\mathbb{Q}\left[\sqrt{p}: p \leq Y\right.$, premier]. Le degré $\left[\mathbb{K}_{Y}: \mathbb{Q}\right]$ est $2^{\pi(Y)}<2^{Y-2}$. Soit $D=\prod_{p<Y} p^{3}<e^{4 Y} / 2$. Comme

$$
D \sum_{1<m_{i} \leq Y} \beta_{i} \leq D(\zeta(3 / 2)-1)<2 D<e^{4 Y},
$$

et comme chacun des nombres $D \beta_{i}$ est un entier algébrique, il est facile de voir que

$$
\begin{aligned}
r(\mathbf{h})^{2^{Y}-2}\left\langle h_{1} \beta_{1}+\ldots+h_{j} \beta_{j}\right\rangle & \geq r(\mathbf{h})^{\left[\mathbb{K}_{Y}: \mathbb{Q}\right]-1}\left\langle h_{1} \beta_{1}+\ldots+h_{j} \beta_{j}\right\rangle \\
& \geq(2 D)^{-\left[\mathbb{K}_{Y}: \mathbb{Q}\right]} \geq e^{-Y 2^{Y}} .
\end{aligned}
$$

Il découle alors immédiatement que pour un point à coordonnées entières $\mathbf{n}$ dans $\mathbb{R}^{j}$, nous avons

$$
\begin{aligned}
& \sum_{\substack{\mathbf{h} \neq 0 \\
\left|h_{i}\right| \leq n_{i}}}\left\langle h_{1} \beta_{1}+\ldots+h_{j} \beta_{j}\right\rangle^{-1} \leq e^{Y 2^{Y}} \prod_{i=1}^{j}\left(\sum_{|h| \leq n_{i}} \max \left\{|h|^{2^{Y}-2}, 1\right\}\right) \\
& \leq e^{Y 2^{Y}} \cdot 2^{j} \cdot \prod_{i=1}^{j} n_{i}^{2^{Y}-1}<e^{Y 2^{Y+1}} r(\mathbf{n})^{2^{Y}-1} .
\end{aligned}
$$

$$
\begin{aligned}
& \sum_{0<\|\mathbf{h}\| \leq M} \frac{1}{r(\mathbf{h})}\left|\frac{1}{N} \sum_{n=1}^{N} e^{2 \pi i\left\langle\mathbf{h}, \mathbf{x}_{n}\right\rangle}\right| \\
& \quad \ll \frac{1}{N} \sum_{0<\|\mathbf{h}\|<M} r^{-1}(\mathbf{h})\left\langle h_{1} \beta_{1}+\ldots+h_{j} \beta_{j}\right\rangle^{-1} \\
& \quad \ll \frac{1}{N} \sum_{n_{1}, \ldots, n_{j}=1}^{M} f\left(n_{1}, \ldots, n_{j}\right) \sum_{\substack{\mathbf{h}=\left(h_{1}, \ldots, h_{j}\right) \\
\left|h_{i}\right| \leq n_{i}}}\left\langle h_{1} \beta_{1}+\ldots+h_{j} \beta_{j}\right\rangle^{-1} \\
& \ll e^{Y 2^{Y+1}} M^{2^{Y}-1},
\end{aligned}
$$


où pour chaque entier $n \leq M$, on a posé $f\left(n_{1}, \ldots, n_{j}\right)=\prod_{i=1}^{j} g\left(n_{i}\right)$, avec

$$
g(n)= \begin{cases}\frac{1}{n(n+1)} & \text { si } n<M, \\ \frac{1}{M} & \text { si } n=M\end{cases}
$$

(voir à cet effet l'exercice \#3.15 de Kuipers et Niederreiter [2]). En additonnant les inégalités (21) pour tous les $j$ et $1<m_{1}<\ldots<m_{j} \leq Y$, avec $m_{i}$ libres de carrés, et en faisant appel à l'inégalité (17), on obtient

$$
\begin{aligned}
& \sum_{j \geq 1} \sum_{\substack{1<m_{1}<\ldots<m_{j} \leq Y \\
\mu\left(m_{i}\right) \neq 0, i=1, \ldots, j}} N D_{\beta_{1}, \ldots, \beta_{j}, N}\left(\mathbf{x}_{1}, \ldots, \mathbf{x}_{N}\right) \\
& \quad \ll 2^{\pi(Y)} \cdot 2 Y^{2} \cdot 3^{Y+1}\left(N / M+e^{Y 2^{Y+1}} M^{2^{Y}-1}\right) \ll e^{Y 2^{Y+2}}\left(N / M+M^{2^{Y}-1}\right) .
\end{aligned}
$$

En posant maintenant $M=\left\lfloor N^{1 / 2^{Y}}\right\rfloor$, on peut alors écrire que

$$
\sum_{j \geq 1} \sum_{\substack{1<m_{1}<\ldots<m_{j} \leq Y \\ \mu\left(m_{i}\right) \neq 0, i=1, \ldots, j}} N D_{\beta_{1}, \ldots, \beta_{j}, N}\left(\mathbf{x}_{1}, \ldots, \mathbf{x}_{N}\right) \ll e^{Y 2^{Y+2}} N^{1-1 / 2^{Y}} .
$$

En substituant (22) dans (20), on conclut que

$$
V(N)=C_{2} N+O\left(\frac{N}{Y^{1 / 2}}+e^{Y 2^{Y+2}} \frac{N}{N^{1 / 2^{Y}}}\right) .
$$

Nous pouvons maintenant choisir $Y$ en le définissant implicitement par l'équation

$$
\frac{N}{Y^{1 / 2}}=e^{Y 2^{Y+2}} \frac{N}{N^{1 / 2^{Y}}},
$$

laquelle est équivalente à

$$
N^{1 / 2^{Y}}=e^{Y 2^{Y+2}} Y^{1 / 2}
$$

ou encore

$$
\frac{\log N}{2^{Y}}=Y 2^{Y+2}+\frac{1}{2} \log Y
$$

ce qui entraîne que

$$
4 Y 4^{Y}(1+o(1))=\log N,
$$

auquel cas

$$
Y(1+o(1)) \log 4=\log \log N,
$$

de sorte que $Y=C_{3}(1+o(1)) \log \log N$ avec $C_{3}=(\log 4)^{-1}$. En substituant cette valeur de $Y$ dans (23), le théorème est démontré.

Remerciements. Les auteurs tiennent à remercier Igor Shparlinski pour une observation fort précieuse qui a permis de compléter la preuve du théorème 1 . 


\section{Références}

[1] A. Ivić, The Riemann Zeta-Function. Theory and Applications, Dover, Mineola, NY, 2003.

[2] L. Kuipers and H. Niederreiter, Uniform Distribution of Sequences, Wiley, New York, 1974.

[3] G. Ricci, Ricerche aritmetiche sui polinomi, Rend. Circ. Mat. Palermo 57 (1933), 433-475.

Département de mathématiques

Mathematical Institute

Université Laval

UNAM

Québec G1K 7P4, Canada

E-mail: jmdk@mat.ulaval.ca

Ap. Postal 61-3 (Xangari), CP 58089

Morelia, Michoacán, Mexico E-mail: fluca@matmor.unam.mx 